\title{
Diferencial Salarial Público-Privado e Desigualdade de Renda per capita no Brasil *
}

\author{
Pedro H. G. F. Souza \\ Pesquisador - (Ipea) \\ Endereço para contato: Ed. BNDS, sala 1402 - Brasília - DF \\ CEP: 70076-900 - E-mail: pedro.ferreira@ipea.gov.br \\ Marcelo Medeiros \\ Professor - Departamento de Sociologia (Unb) e (Ipea) \\ Endereço para contato: Ed. BNDES, sala 1402 - Brasília - DF \\ CEP: $70076-900$
}

Recebido em 14 de agosto de 2012. Aceito em 25 de novembro de 2012.

\begin{abstract}
Resumo
O estudo estima a contribuição específica do diferencial salarial entre trabalhadores com características semelhantes dos setores público e privado para a desigualdade na renda domiciliar per capita no Brasil. A estimação baseia-se em simulações contrafactuais e o cálculo das contribuições para a desigualdade em uma decomposição de fatores do coeficiente de Gini. Os dados são provenientes da PNAD 2009. O diferencial equivale a cerca de $17 \%$ da massa salarial dos servidores públicos, é regressivo e altamente concentrado, mas tem volume pequeno na renda total $(1 \%)$ e, por isso, contribui para cerca de $3 \%$ da desigualdade total. Os efeitos sobre a desigualdade da composição da força de trabalho nos setores são muito maiores que efeitos da segmentação do mercado de trabalho entre os dois setores. Essas conclusões são pouco sensíveis a modificações de definições de setor e diferentes técnicas de estimação.
\end{abstract}

\section{Palavras-Chave}

desigualdade de renda, desigualdade salarial, funcionários públicos, diferenciais salariais, segmentação do mercado de trabalho

\begin{abstract}
We estimate the contribution of the wage differential between workers with the same attributes in the public and private sectors to the household per capita income inequality in Brazil. The estimate is based on counterfactual simulations and the contribution to inequality on a factor decomposition of the Gini coefficient. Data comes from the Brazilian National Household Survey PNAD 2009. The differential corresponds approximately to $17 \%$ of the wage bill of workers in the public sector, is regressive and highly concentrated. However, because it amounts to a small share of the total income $(1 \%)$ its contribution to the total inequality is of $3 \%$. The sector composition effects on inequality are times higher than the segmentation (price) effects. These conclusions are robust to changes in the definition of the sectors and to different estimation techniques.
\end{abstract}

- Os autores agradecem a Sergei Soares, Ana Luiza Neves de Holanda Barbosa e Fabio Veras Soares pelos comentários e sugestões. 


\section{Keywords}

income inequality, wage inequality, public sector workers, wage differentials, labor market segmentation

JEL Classification

D63, E24, J31, J45, J42,

\section{Introdução}

O objetivo deste trabalho é estimar a contribuição específica do diferencial público-privado para a desigualdade na renda domiciliar per capita no Brasil. O diferencial salarial entre os setores público e privado é de especial interesse para o estudo da desigualdade, pois indica em que medida o Estado reforça ou reduz desigualdades em algumas de suas ações. O diferencial é estimado por meio da metodologia proposta por Juhn, Murphy e Pierce (1993) e as contribuições para a desigualdade são calculadas a partir da decomposição de fatores do coeficiente de Gini desenvolvida por Rao (1969) e disseminada por Shorrocks (1982). Os resultados indicam que a vantagem salarial dos empregados públicos contribui para cerca de $3 \%$ da desigualdade total.

Em termos mais gerais, o que este estudo tem por objeto é um mecanismo importante que o Estado possui para afetar diretamente os níveis de desigualdade no país, mas que não recebeu ainda maiores atenções na literatura sobre o assunto. A segmentação do mercado de trabalho entre setores público e privado abre a possibilidade de que haja componentes fortemente institucionais na determinação da desigualdade total e a estimativa da contribuição dos diferenciais salariais para a desigualdade em certa medida isola um desses componentes, a demanda por mão de obra, que é afetada pelas políticas salariais do Estado em seus diversos níveis.

As políticas salariais estatais são influenciadas, por um lado, pelo funcionamento do mercado geral de trabalho, pois o Estado compete por mão de obra nesse mercado. Por outro, há determinantes políticos importantes na determinação dos salários dos trabalhadores do setor público, uma vez que estes também são agentes políticos e compõem um corpo eleitoral de grandes proporções. Finalmente, o setor público é um segmento do mercado de trabalho regulado 
por regras bastante particulares e que visa objetivos distintos dos do setor privado. As distinções entre os dois setores abrem espaço para a emergência de diferenças nos salários pagos a trabalhadores equivalentes em cada setor.

Assim como ocorre em alguns países, no Brasil os empregados do setor público hoje tendem a ter rendimentos superiores aos dos trabalhadores do setor privado, mesmo quando se comparam trabalhadores com atributos semelhantes. Nos anos 1970, o diferencial entre os trabalhadores dos dois setores indicava desvantagem dos empregados públicos, mas, a partir de meados da década de 1990, a desvantagem foi revertida e o prêmio salarial destes últimos vem aumentando. A vantagem atual dos empregados públicos tende a ser maior entre aqueles com menor nível educacional, os que trabalham para a União e os que trabalham em atividades do setor jurídico (Belluzzo, Anuatti-Neto, \& Pazello, 2005; Bender \& Fernandes, 2009; Braga, 2007; Foguel, Gill, Mendonça, \& Barros, 2000; Gregory \& Borland, 1999; Panizza \& Qiang, 2005; Vaz \& Hoffmann, 2007; Vergara, 1991; Vergara \& da Silva Wiltgen, 1995)

Não se sabe qual a contribuição do diferencial para a desigualdade total, mas o tamanho e a composição do setor público e o seu perfil salarial são relativamente bem conhecidos. Este setor tem a peculiaridade de ser composto, predominantemente, por trabalhadores com qualificação superior à do restante da força de trabalho e que, por esta razão, geralmente pertencem aos estratos superiores da sociedade. De fato, observa-se que embora o número de empregados civis e militares no Brasil seja relativamente pequeno, sua massa salarial total não é desprezível, possui uma distribuição muito concentrada e contribui com uma fração relevante da desigualdade de rendimentos (Hoffmann, 2009). Nosso estudo dialoga com os resultados de Hoffmann à medida que desagrega da massa de salários o diferencial salarial entre os setores público e privado.

A atual vantagem dos empregados públicos pode ser atribuída a dois conjuntos de fatores: os trabalhadores do setor público têm mais educação e por isso podem ocupar postos que requerem maior qualificação; além disso, a segmentação do mercado de trabalho permite que o setor público estabeleça salários mais altos que os de trabalhadores equivalentes no setor privado. O primeiro conjunto pode ser associado a um efeito de composição da força de trabalho em cada 
setor, denominado simplesmente efeito composição, ao passo que o segundo reflete diferenças nas regras de determinação de salários para trabalhadores com características semelhantes, denominado aqui efeito segmentação, mas também conhecido como efeito preços.

As vantagens salariais do segmento público podem estar associadas ao fato de que Estado tem objetivos distintos dos perseguidos por empresas privadas, em particular no que diz respeito a metas de redução de custos de mão de obra para aumentar lucros. Por um lado, os administradores do setor público sofrem influências diretas de objetivos políticos e tendem a tratar a política salarial do Estado como meio para alcançar esses objetivos. Colocado de modo direto, políticos sabem que maiores salários podem implicar apoio na condução de suas propostas políticas ou, até mesmo, alinhamento eleitoral. Por outro lado, as várias categorias de empregados públicos formam blocos grandes, relativamente uniformes e com um único contratador, o que facilita a organização de sindicatos fortes para a defesa de seus salários (Gregory \& Borland, 1999). Evidência dessa capacidade de mobilização no Brasil é dada pelo fato de que, no setor público, carreiras distintas são tratadas por políticas salariais muito homogêneas (Marconi, 2003).

Regras de remuneração distintas em cada segmento do mercado de trabalho fazem com que exista um diferencial salarial total que equivale à agregação dos hiatos entre o que é efetivamente pago em um setor para um trabalhador com determinadas características e o que seria pago a esse mesmo trabalhador se ele fosse remunerado pelas regras do outro setor. Tomando-se o setor público como referência, o diferencial salarial total corresponde à soma das diferenças entre as remunerações observadas dos empregados no setor público e as remunerações estimadas para esses trabalhadores caso estivessem empregados no setor privado. Parte da desigualdade total na distribuição de renda pode ser atribuída a esse diferencial. O foco no diferencial faz com que o efeito composição da força de trabalho nos dois setores seja, na medida do possível, controlado, e a contribuição para a desigualdade do efeito segmentação, isolada. 


\section{Definições e Metodologia}

\subsection{Dados}

Os dados utilizados são provenientes da Pesquisa Nacional por Amostra de Domicílios de 2009 (PNAD 2009), realizada pelo do Instituto Brasileiro de Geografia e Estatística (IBGE), cuja amostra é desenhada para representar praticamente toda a população do Brasil. Foram excluídos da análise os indivíduos com renda domiciliar per capita ignorada e os indivíduos cuja condição no domicílio era de pensionista, empregado doméstico ou parente de empregado doméstico (grupo distinto dos trabalhadores domésticos que não residem no domicílio dos empregadores). A amostra analisada soma 386.728 pessoas em 117.827 domicílios, a qual, expandida, corresponde a uma população de 185,1 milhões de pessoas em 56,9 milhões de domicílios. Toda população com rendimentos válidos foi considerada no estudo. Para os cálculos de diferenciais salariais, apenas a população ocupada e com idade igual ou superior a 18 anos foi analisada, o que significa 163.084 indivíduos representando uma população de 78,4 milhões de pessoas. Esse grupo, por sua vez, foi subdividido e as populações equivalentes às subdivisões apresentadas na Tabela 1.

No estudo as distribuições de renda tratam apenas de rendimentos monetários no mês de referência da PNAD 2009 (setembro), incluindo-se não apenas salários, mas também outras rendas que não as do trabalho, tais como rendimentos correntes de aposentadorias e pensões, rendimentos de capital, aluguéis, programas sociais, doações em espécie, etc. Não são computados quaisquer tipos de rendimentos não monetários ou vantagens funcionais, tampouco são levadas em contas diferenças de longo prazo nos rendimentos que podem ser associadas à segmentação do mercado de trabalho, como as causadas pela diferenciação dos regimes de aposentadoria público e privado.

\subsection{Definições e Análise de Sensibilidade}

Há distintas maneiras de se definir trabalhadores do setor público e empregados comparáveis do setor privado, bem como procedimentos de cálculo dos diferenciais salariais (Holanda, 2009). Como não existe consenso nos estudos brasileiros sobre a metodologia mais 
adequada a usar, a robustez dos resultados foi avaliada por meio de uma análise de sensibilidade que varia tanto definições quanto procedimentos. Duas definições de setor público e setor privado comparável e quatro procedimentos de cálculo dos diferenciais foram testados, seus detalhes sendo discutidos abaixo. Algumas variações metodológicas têm impactos sobre os valores estimados de contribuição para a desigualdade, mas não afetam de modo radical as conclusões substantivas.

$\mathrm{Na}$ definição denominada abrangente, são considerados empregados do setor público todas as pessoas de 18 anos ou mais que em seu trabalho principal são funcionários estatutários civis ou militares, qualquer que seja o nível de governo, e definidos como empregados comparáveis do setor privado todos os empregados de 18 anos ou mais com carteira de trabalho assinada na ocupação principal. Os trabalhadores restantes - empregadores, empregados por conta própria, empregados sem carteira, trabalhadores com idades distintas, etc., são classificados como demais trabalhadores e mantidos na análise da desigualdade, mas não são considerados nos cálculos dos diferenciais salariais.

$\mathrm{Na}$ definição restrita exclui-se do setor público os militares e, dos empregados comparáveis do setor privado, os empregados domésticos e os trabalhadores do setor agrícola. Ou seja, passam a ser considerados empregados do setor público apenas as pessoas de 18 anos ou mais que em seu trabalho principal são funcionários estatutários civis e definidos como empregados comparáveis do setor privado os empregados com carteira de trabalho assinada que não trabalhavam nos setores agrícola e doméstico. Nesta segunda definição, ainda que a segmentação do mercado de trabalho persista, os grupos comparados são mais homogêneos no que diz respeito a atributos pessoais dos trabalhadores, critérios de remuneração, forma de organização do trabalho, natureza da atividade e representação política. Para o cálculo de diferenciais salariais foram testados três tipos de procedimentos: regressão log-lin de equação de salários em estágio único, sem correção para viés de seleção; regressão em dois estágios, com correção para viés de seleção que modela o direcionamento a um setor de atividade (público ou privado); e regressão em dois estágios, com correção para viés de seleção a partir de um probit bivariado que modela simultaneamente a probabilidade de se trabalhar ou não e o direcionamento a um setor de atividade. 
A combinação das duas definições e dos três tipos de procedimentos resultou em seis simulações distintas. Além disso, no caso da definição restrita, foram realizadas outras três simulações que simplesmente acrescentaram à equação de estimação da renda contrafactual dos três tipos de procedimentos uma variável categórica relativa ao grupo ocupacional do indivíduo, cujo objetivo é o de controlar os efeitos sobre os salários causados por diferenças nas formas de organização burocrática do trabalho nos setores público e privado. Todas as simulações geraram resultados bastante consistentes entre si e, por isso, apresentam-se abaixo apenas os resultados relativos aos modelos mais simples (definição abrangente, sem correção, sem variável ocupacional) e mais complexo (definição restrita, com correção via probit bivariado, com variável ocupacional).

\subsection{Estimativas do Diferencial Salarial}

A metodologia para mensurar os efeitos do diferencial salarial entre os empregados dos setores público e privado na desigualdade de renda domiciliar per capita consiste em dois passos: i) estimação da remuneração contrafactual que cada empregado do setor público receberia caso trabalhasse no setor privado formal; ii) cálculo do diferencial salarial para cada empregado do setor público. O Anexo 1 traz a formalização dos procedimentos utilizados nesta seção.

A renda contrafactual é estimada por meio da decomposição proposta por Juhn, Murphy e Pierce (JMP), a qual permite isolar os efeitos preço, quantidade e efeitos não explicados por regressões lineares (Juhn et al., 1993). Estima-se, primeiro, uma equação de rendimentos para um grupo de referência e, em seguida, estima-se a mesma equação para um grupo alternativo. A renda simulada do grupo de referência é calculada como sendo a renda obtida por esse grupo caso os preços (coeficientes) e a distribuição dos efeitos não explicados (resíduo) fossem, para esse grupo, equivalentes aos do grupo alternativo - ou seja, permite-se apenas a variação das quantidades (atributos pessoais e outros). Aqui, o grupo de referência é o conjunto de funcionários públicos e o grupo alternativo, os empregados comparáveis do setor privado. A equação de salários usada como base tem como variável dependente o logaritmo do rendimento mensal do trabalho principal. Os rendimentos derivados de trabalhos 
secundários foram desconsiderados e agregados aos "demais rendimentos", utilizados apenas para calcular o efeito sobre a desigualdade na renda domiciliar per capita. O Quadro 1 traz a lista de variáveis independentes:

Quadro 1 - Variáveis independentes do modelo básico de equação de salários

\begin{tabular}{|c|c|}
\hline Nome & Descrição \\
\hline Educação & $\begin{array}{l}\text { Nove variáveis dummy para ensino elementar incompleto, ensino elementar completo, ensino } \\
\text { fundamental incompleto, ensino fundamental completo, ensino médio incompleto, ensino médio } \\
\text { completo, ensino superior incompleto, ensino superior completo, pós-graduação completa ou } \\
\text { incompleta e, como base, os analfabetos. }\end{array}$ \\
\hline Cor ou raça & Variável dummy para brancos ou amarelos e, como base, todos os demais grupos; \\
\hline Sexo & Variável dummy para homens e, como base, mulheres; \\
\hline Experiência & $\begin{array}{l}\text { Experiência em anos no mercado de trabalho normalizada - para diminuir possíveis problemas de } \\
\text { multicolinearidade - para ter média igual a zero e desvio padrão igual a um, e o seu quadrado; }\end{array}$ \\
\hline Tempo na ocupação & $\begin{array}{l}\text { Tempo em anos na ocupação atual normalizado - para diminuir possíveis problemas de } \\
\text { multicolinearidade - para ter média igual a zero e desvio padrão igual a um, e o seu quadrado; }\end{array}$ \\
\hline Jornada & Logaritmo do número de horas trabalhadas por semana na ocupação principal; \\
\hline Macrorregião & $\begin{array}{l}\text { Quatro variáveis dummy para regiões Nordeste, Sudeste, Sul e Centro-Oeste, tendo a região } \\
\text { Norte como referência; }\end{array}$ \\
\hline Área de residência & Variável dummy para áreas urbanas, tendo as áreas rurais como referência; \\
\hline Tipo de município & $\begin{array}{l}\text { Duas variáveis dummy para e municípios não metropolitanos de grande porte (municípios } \\
\text { autorrepresentativos, de acordo com o desenho amostral da PNAD) e municípios de pequeno } \\
\text { porte (municípios não autorrepresentativos na PNAD), tendo os municípios em regiões } \\
\text { metropolitanas como referência; }\end{array}$ \\
\hline
\end{tabular}

À equação base foram acrescentadas variáveis de modo a criar um segundo modelo de regressão. $\mathrm{O}$ acréscimo visa controlar efeitos de heterogeneidade nos grupos ocupacionais entre os setores público e privado por intermédio de quatro variáveis dummy para profissionais das ciências e das artes, técnicos de nível médio/trabalhadores de serviços administrativos, trabalhadores dos serviços e demais profissionais, tendo os dirigentes como categoria de referência, e corrigir viés de seleção por meio de duas variáveis da razão inversa de Mills, calculadas a partir das probabilidades estimadas por um probit bivariado.

No caso da simulação com correção simples para viés de seleção, a variável dependente foi o setor do trabalho principal (público ou privado); no caso do probit bivariado, as duas variáveis dependentes foram a participação ou não no mercado de trabalho, remunerado o direcionamento a um setor de atividade. Em todos os casos, as equações de seleção foram aplicadas somente a indivíduos de 18 anos ou 
mais. As variáveis independentes dos probits foram: variáveis para área de residência, tipo de município, cor e escolaridade idênticas às descritas acima; 26 variáveis dummy para Unidade da Federação (Rondônia como base); polinômio para idade - normalizada - e o seu quadrado; quatro variáveis dummy para posição no domicílio (chefe do domicílio como referência); dummy para presença de crianças de até 15 anos no domicílio; dummy para frequência à escola; e duas variáveis dummy para o setor de trabalho do cônjuge (não tem cônjuge/ cônjuge não trabalha como referência).

As estimativas de diferencial salarial são calculadas apenas para o rendimento do trabalho principal de trabalhadores, contrastando empregados públicos e privados comparáveis. As contribuições do diferencial estimado para a desigualdade salarial total e para a desigualdade total da renda per capita, porém, foram calculadas levando-se em conta todos os rendimentos de todos os trabalhadores, isto é, para todas as idades, incluindo os rendimentos dos trabalhos secundários e incorporando os demais trabalhadores.

O diferencial salarial corresponde à diferença entre os rendimentos observado e simulado. Esse diferencial pode ser dividido em dois fatores da distribuição de renda, a saber, diferencial positivo, o qual ocorre quando os funcionários públicos recebem mais que seus equivalentes privados - ou seja, têm um prêmio salarial - e diferencial negativo, no caso de desvantagens relativas. A existência de um fator negativo tem implicações para a decomposição da desigualdade, como se discute adiante.

\subsection{Decomposição da Desigualdade}

A desigualdade na distribuição dos rendimentos domiciliares per capita, medida pelo coeficiente de Gini, é decomposta em função de seus fatores (fontes de rendimento) a partir de dois parâmetros, o coeficiente de concentração $\left(\mathrm{C}_{\mathrm{h}}\right)$ do fator, a participação relativa desse fator no total de rendimentos $\left(\varphi_{\mathrm{h}}\right)$. A contribuição de cada fator para a desigualdade total é dada pelo produto dos dois parâmetros $\left(\mathrm{C}_{\mathrm{h}} \cdot \varphi_{\mathrm{h}}\right)$. A soma das contribuições de cada fator equivale ao coeficiente de Gini (Rao, 1969; Shorrocks, 1982). O índice de progressividade de cada fator, $\left(\pi_{\mathrm{h}}\right)$, é calculado pela diferença entre o índice de Gini e o coeficiente de concentração do fator $\left(\pi_{h}=G-C_{h}\right)$. 
Esse índice relaciona a concentração de um fator específico com a concentração da renda total, podendo ter valores positivos, que indicam que o fator, ainda que esteja desigualmente distribuído, contribui para reduzir a desigualdade total, ou negativos, indicando o contrário. O Anexo 1 traz a formalização dessa decomposição.

Neste trabalho, a renda observada para os funcionários públicos foi dividida em três fatores: a renda simulada, o diferencial positivo e o diferencial negativo. Ou seja, decomposição por fatores da distribuição de renda domiciliar per capita trata o diferencial estimado como um desses fatores, como se fosse um rendimento adicionado ou subtraído dos salários dos empregados do setor público. Uma decomposição aditiva com coeficientes de concentração dentro do limite convencional, -1 e +1 , é assegurada quando apenas fatores com valores positivos são considerados (Chen, Tsaur, \& Rhai, 1982; Rao, 1969). Quando um mesmo fator possui rendas negativas e positivas, existe a possibilidade dos coeficientes de concentração se situarem fora da escala -1 e +1 (Pyatt, Chen, \& Fei, 1980). Isso ocorre quando o cálculo do coeficiente de concentração relaciona uma curva de concentração com valores negativos a uma curva de Lorenz somente com valores positivos. Há três alternativas para lidar com esta particularidade. A primeira é ajustar a desigualdade observada no coeficiente de Gini por meio da inclusão de valores negativos hipotéticos na área da curva de Lorenz observada. Isso força os coeficientes de concentração para dentro dos limites -1 e +1 , mas provoca uma redução artificial dos níveis de desigualdade mensurados quando comparados aos níveis convencionalmente medidos. A segunda é dividir o fator com valores negativos e positivos em dois subfatores, um formado apenas pelos valores negativos e outro apenas pelos valores positivos. Cada subfator terá coeficientes de concentração variando dentro da escala convencional e o nível de desigualdade observado se manterá. A terceira é não dividir o fator com valores positivos e negativos e aceitar coeficientes de concentração fora da escala convencional, o que tem a vantagem de não modificar artificialmente os níveis de desigualdade, mas requer uma interpretação distinta do nível de concentração do fator que contém rendas negativas.

Como o objeto principal deste estudo é obter a contribuição relativa dos fatores para a desigualdade total observada, as opções adotadas foram as duas últimas, ou seja, dividir o fator diferencial salarial total em subfatores diferencial positivo e diferencial negativo para o 
cálculo de suas concentrações, progressividade e contribuições isoladas para a desigualdade, e, no caso do diferencial salarial total, aceitar um coeficiente de concentração variando fora da escala convencional. Note-se que esta última opção não implica um cálculo errôneo do coeficiente de concentração, mas sim do uso de uma escala diferente de variação desse coeficiente.

\section{Resultados}

Somados, os dois grupos comparados correspondem a cerca de metade da força de trabalho ocupada no Brasil. Os trabalhadores do setor público correspondem a pouco menos de um décimo; seus equivalentes, trabalhadores formais do setor privado, a pouco mais de $40 \%$ dos ocupados. A metade restante é formada por todas as demais categorias de ocupados com idade superior a 18 anos. A Tabela 1 mostra que a definição mais restrita de funcionários públicos exclui 0,3 milhões de militares, o que corresponde a menos de um vigésimo dos funcionários públicos. A restrição de setores aplicada entre os trabalhadores privados formais é de magnitude muito maior, 3,5 milhões de pessoas, mas que, proporcionalmente, não é tão grande, representando algo em torno de um décimo dessa categoria de trabalhadores.

Tabela 1 - Estatísticas descritivas dos subgrupos analisados, Brasil, 2009

\begin{tabular}{|c|c|c|c|c|c|}
\hline População & $\begin{array}{c}\mathrm{N} \\
\text { (milhões) }\end{array}$ & $\%$ & $\begin{array}{c}\text { Renda } \\
\text { trabalho } \\
\text { Média }\end{array}$ & Gini & $\begin{array}{l}\text { Jornada } \\
\text { semanal } \\
\text { (horas) }\end{array}$ \\
\hline Funcionários públicos (definição abrangente) & 6,4 & 8.1 & 1916 & .481 & 37.4 \\
\hline Funcionários públicos (definição restrita) & 6,1 & 7.8 & 1906 & .482 & 37.2 \\
\hline Militares & 0,3 & 0.3 & 2144 & .438 & 42.4 \\
\hline Ocupados formais do setor privado (definição abrangente) & 32,7 & 41.7 & 1073 & .385 & 43.8 \\
\hline Ocupados formais do setor privado (definição restrita) & 29,2 & 37.2 & 1125 & .390 & 43.6 \\
\hline Ocupados formais do setor privado (setores excluídos) & 3,5 & 4.5 & 644 & .204 & 45.7 \\
\hline Demais trabalhadores & 39.4 & 50.3 & 950 & .571 & 39.5 \\
\hline Trabalhadores ocupados remunerados (total) & 78.4 & 100 & 1080 & .501 & 41.1 \\
\hline
\end{tabular}

Fonte: PNAD 2009.

Notas: Renda refere-se ao rendimento mensal do trabalho principal em reais de setembro de 2009; a população analisada exclui pessoas cuja condição no domicílio era de agregado, pensionista, empregado doméstico e filho de empregado doméstico, ou com menos de 18 anos de idade; os setores excluídos na definição restrita dos ocupados formais do setor privado são agricultura e serviços domésticos. 
Os funcionários públicos têm rendimentos médios bem superiores aos dos demais trabalhadores e a exclusão dos militares da categoria não altera expressivamente esse quadro. Os ocupados formais do setor privado têm salários médios próximos dos salários dos demais trabalhadores. A exclusão de setores na definição de ocupados formais privados comparáveis aumenta os salários médios do grupo, mas ainda assim permanece uma diferença substantiva em relação ao setor público. Essas diferenças entre médias, porém, devem ser tratadas com cautela. O simples fato de pertencer ao setor público não assegura remunerações maiores a um trabalhador. As desigualdades internas de cada categoria são muito grandes, com coeficientes de Gini bem acima de 0,390 em quase todos os grupos comparados. Desigualdades salariais baixas são observadas apenas entre os ocupados formais domésticos e na agricultura, cujos rendimentos são notadamente mais baixos que os de todas as demais categorias de trabalhadores.

A mensuração dos efeitos do diferencial salarial entre os empregados dos setores público e privado na desigualdade de renda domiciliar per capita depende de uma estimação da remuneração que um empregado do setor público receberia se trabalhasse no setor privado formal. A Tabela 2 apresenta resultados que permitem avaliar a sensibilidade de diferentes especificações de modelos usados para essa estimação. São comparados modelos que utilizam as duas definições de setor público e privado (abrangente e restrita), distintas formas de modelar a correção de viés de amostra (nenhuma, simples, biprobit) e uso de variáveis de controle de grupos ocupacionais nas equações de rendimentos. 
Tabela 2 - Sensibilidade de médias e distribuições dos rendimentos do trabalho principal dos trabalhadores do setor público, segundo tipos de modelos utilizados - Brasil, 2009

\begin{tabular}{|c|c|c|c|c|c|c|}
\hline Definição & $\begin{array}{l}\text { Tipo de } \\
\text { correção }\end{array}$ & $\begin{array}{l}\text { Modelo de } \\
\text { regressão }\end{array}$ & $\begin{array}{l}\text { Renda do } \\
\text { trabalho média } \\
\text { simulada }\end{array}$ & $\begin{array}{c}\text { Renda do trabalho } \\
\text { observada/simula- } \\
\text { da (\%) }\end{array}$ & $\begin{array}{l}\text { Gini da renda } \\
\text { do trabalho } \\
\text { simulada }\end{array}$ & $\begin{array}{c}\text { Gini renda } \\
\text { observado/simulado } \\
(\%)\end{array}$ \\
\hline Abrangente & Nenhuma & $\begin{array}{l}\text { Sem grupos } \\
\text { ocupacionais }\end{array}$ & 1580 & 121 & 0.408 & 118 \\
\hline Abrangente & Simples & $\begin{array}{l}\text { Sem grupos } \\
\text { ocupacionais }\end{array}$ & 1597 & 120 & 0.409 & 118 \\
\hline Abrangente & Biprobit & $\begin{array}{l}\text { Sem grupos } \\
\text { ocupacionais }\end{array}$ & 1580 & 121 & 0.407 & 118 \\
\hline Restrita & Nenhuma & $\begin{array}{l}\text { Sem grupos } \\
\text { ocupacionais }\end{array}$ & 1651 & 115 & 0.413 & 117 \\
\hline Restrita & Nenhuma & $\begin{array}{l}\text { Com grupos } \\
\text { ocupacionais }\end{array}$ & 1649 & 116 & 0.410 & 118 \\
\hline Restrita & Simples & $\begin{array}{l}\text { Sem grupos } \\
\text { ocupacionais }\end{array}$ & 1645 & 116 & 0.412 & 117 \\
\hline Restrita & Simples & $\begin{array}{l}\text { Com grupos } \\
\text { ocupacionais }\end{array}$ & 1644 & 116 & 0.410 & 118 \\
\hline Restrita & Biprobit & $\begin{array}{l}\text { Sem grupos } \\
\text { ocupacionais }\end{array}$ & 1644 & 116 & 0.411 & 117 \\
\hline Restrita & Biprobit & $\begin{array}{l}\text { Com grupos } \\
\text { ocupacionais }\end{array}$ & 1653 & 115 & 0.409 & 118 \\
\hline
\end{tabular}

Fonte: PNAD 2009.

Notas: "Renda do trabalho" refere-se ao rendimento mensal do trabalho principal em reais de setembro de 2009; a população analisada exclui pessoas cuja condição no domicílio era de agregado, pensionista, empregado doméstico e filho de empregado doméstico, ou com menos de 18 anos de idade; os setores excluídos na definição restrita dos ocupados formais do setor privado são agricultura e serviços domésticos. Os grupos ocupacionais controlados são profissionais das ciências e das artes, técnicos de nível médio e trabalhadores de serviços administrativos, trabalhadores dos serviços e profissionais.

As simulações são robustas a variações na especificação dos modelos e o conteúdo substantivo da análise não é expressivamente alterado pelo uso de variáveis de controle de ocupação, nem pela metodologia de correção de viés. A razão entre a renda do trabalho observada e simulada e a razão entre os coeficientes de Gini das distribuições de renda domiciliar per capita observadas e simuladas praticamente não são alterados quando as especificações dos modelos de simulação são modificadas.

Em todas as nove simulações, o diferencial médio fica entre 15\% e $21 \%$, ou seja, os funcionários públicos ganham cerca de um quinto a mais do que trabalhadores equivalentes do setor privado. Por exemplo, no modelo mais complexo, com definição restrita e correção via probit, a renda média simulada é de $\mathrm{R} \$ 1653$, contra uma renda 
média observada de $\mathrm{R} \$ 1906$. Além da menor renda média, as simulações também geram distribuições de renda menos desiguais entre funcionários públicos: o Gini observado para esse grupo é sempre $17 \%$ ou $18 \%$ maior do que o simulado. Ou seja, o diferencial salarial não beneficia de forma homogênea todos os funcionários públicos, estando concentrado nos membros mais ricos desse grupo.

Para análises de desigualdade, o que em alguma medida importa são as definições do que constitui o setor público e seu equivalente privado. Ou seja, há algumas diferenças nos resultados se militares são excluídos do conjunto dos funcionários públicos e os trabalhadores nos setores agricultura e doméstico são excluídos do conjunto dos trabalhadores formais do setor privado. Todavia, mesmo essas diferenças são pequenas, limitadas a variações de seis pontos percentuais nas razões de renda observada e simulada e flutuações sem relevância nos coeficientes de Gini. A interpretação substantiva deste estudo, portanto, não deve ser alterada em função da escolha por uma das alternativas metodológicas elencadas, razão pela qual as estimativas das contribuições dos diferenciais salariais para a desigualdade de renda domiciliar são apresentadas na Tabela 3 levando-se em conta apenas a maior e a menor estimativa.

A Tabela 3 apresenta a decomposição de fatores (fontes de rendimento) da desigualdade em distribuições geradas em duas simulações, a saber, a que usa a definição mais abrangente e a metodologia mais simples e produz os menores rendimentos médios do trabalho, e a que usa a definição restrita e maior complexidade metodológica, tendo como resultado os rendimentos médios mais altos. Essa tabela decompõe ainda o diferencial entre setores público e privado em diferencial positivo, para os trabalhadores do setor público, que, conforme simulado, ganham mais do que ganhariam se trabalhassem no setor privado, e diferencial negativo, no caso contrário. Este último equivale a uma perda salarial e, portanto, aparece como uma renda negativa. Seu coeficiente de concentração indica a distribuição dessa perda nos diferentes estratos sociais. 
Tabela 3 - Decomposição da desigualdade na renda domiciliar per capita segundo fatores - Brasil, 2009

\begin{tabular}{lrrrrr}
\hline Fator & $\begin{array}{l}\text { Coeficiente } \\
\text { Concentração }\end{array}$ & $\begin{array}{c}\text { \% Renda } \\
\text { domiciliar }\end{array}$ & $\begin{array}{l}\text { Contribuição } \\
\text { Gini }\end{array}$ & $\begin{array}{l}\text { \% Gini renda } \\
\text { domiciliar }\end{array}$ & Progressividade \\
\hline Emp. Formais privados, observado & 0,481 & 30,5 & 0,144 & 26,7 & 0,059 \\
Emp. públicos, simulado & 0,703 & 8,6 & 0,061 & 11,2 & $-0,163$ \\
Dif. público-privado total & $0,916^{*}$ & 1,8 & 0,017 & 3,1 & $-0,376^{*}$ \\
Dif. público-privado positivo & 0,852 & 2,1 & 0,018 & 3,4 & $-0,312$ \\
Dif. público-privado negativo & 0,470 & 0,3 & $-0,001$ & $-0,3$ & 0,070 \\
Todas outras rendas & 0,534 & 59,5 & 0,318 & 59,0 & 0,005 \\
2. Definição restrita, com correção via biprobit, com variável ocupacional & & & \\
Emp. Formais privados, observado & 0,509 & 28,1 & 0,143 & 26,4 & 0,031 \\
Emp. públicos, simulado & 0,701 & 8,6 & 0,061 & 11,2 & $-0,161$ \\
Dif. público-privado total & $1,003^{*}$ & 1,4 & 0,013 & 2,5 & $-0,463^{*}$ \\
Dif. público-privado positivo & 0,863 & 1,9 & 0,016 & 3,0 & $-0,323$ \\
Dif. público-privado negativo & 0,518 & $-0,5$ & $-0,003$ & $-0,5$ & 0,022 \\
Todas outras rendas & 0,522 & 62,0 & 0,323 & 59,9 & 0,018 \\
\hline Gini & 0,540 & $100 \%$ & 0,540 & $100 \%$ & 0,000 \\
\hline
\end{tabular}

Fonte: PNAD 2009.

* Por usarem uma escala diferente os coeficientes de concentração e índices de progressividade desse fator não são diretamente comparáveis aos dos demais fatores.

As simulações produzem parâmetros levemente distintos, mas levam às mesmas conclusões. O diferencial que existe entre os salários de trabalhadores dos setores público e privado com atributos semelhantes é muito concentrado e regressivo. Sua contribuição para a desigualdade total, todavia, não é grande, pois seu peso no rendimento domiciliar total é pequeno. Muito mais importante para a desigualdade total que esse diferencial, ou até mesmo que o conjunto dos rendimentos dos trabalhadores formais do setor privado e funcionários públicos, é a distribuição de todas as outras fontes de rendimento, inclusive os rendimentos dos trabalhadores informais, aposentadorias e pensões, aluguéis e rendimentos de capital, etc. Há uma segmentação do mercado de trabalho entre os setores público e privado, mas o efeito composição dessa segmentação sobre a desigualdade é muito maior do que o efeito segmentação (efeito preços). 
Em outras palavras, a composição do setor público, com trabalhadores mais qualificados que os do setor privado, tem muito mais peso sobre a desigualdade que os diferenciais salariais associadas à segmentação.

O diferencial salarial, quando positivo, resulta em concentração nos trabalhadores de estratos mais altos da sociedade, tendo coeficientes de concentração de 0,852 e 0,863, a depender da simulação. Trata-se de medidas de concentração bem superiores ao coeficiente de Gini observado na distribuição dos rendimentos domiciliares per capita, que é de 0,540. O diferencial negativo também é concentrado, porém menos até do que o coeficiente de Gini, sendo levemente progressivo e, consequentemente, tende a contribuir um pouco para a redução da desigualdade. A massa do diferencial positivo, no entanto, é várias vezes maior que o do diferencial negativo, fazendo com que o saldo final dos diferenciais aponte para uma vantagem média dos trabalhadores do setor público concentradora, mesmo levando em conta diferenças nas escalas de variação deste fator e os demais fatores.

Mais importante e indiferente à escala utilizada na medida de concentração é que os diferenciais contribuem pouco para a desigualdade na distribuição dos rendimentos domiciliares per capita. Essa contribuição corresponde a $3,1 \%$ e $2,5 \%$ da desigualdade total medida pelo coeficiente de Gini, a depender da estimativa. Isso porque os diferenciais equivalem a apenas $1,8 \%$ e $1,4 \%$ dos rendimentos domiciliares totais. A contribuição de outras fontes com maior peso na renda chega a ser dezena de vezes superior, ainda que essas fontes sejam bem menos concentradas que os diferenciais individuais.

\section{Conclusões}

Existe uma segmentação do mercado de trabalho brasileiro entre os setores público e privado no que diz respeito à determinação dos salários. Tal como ocorre em outros países, no Brasil os empregados do setor público tendem a receber rendimentos superiores aos dos empregados do setor privado. Essa diferença está relacionada a dois fatores. O primeiro é um efeito de composição, que ocorre porque a força de trabalho do setor público é composta por trabalhadores que, por terem maior nível educacional, tendem a receber salários maiores 
que os trabalhadores de menor qualificação, seja qual for o setor a que pertençam. O segundo é um efeito segmentação, cuja implicação para a determinação dos preços do trabalho se manifesta na existência de um diferencial salarial, na média favorável aos trabalhadores públicos, mesmo quando comparados a trabalhadores privados com características similares às suas. No Brasil o efeito composição tem muito mais peso sobre a desigualdade que o efeito segmentação.

O diferencial salarial associado à segmentação público-privado é evidência da atuação de componentes institucionais importantes na determinação das desigualdades salariais no mercado de trabalho. Como essas desigualdades salariais contribuem para a desigualdade de rendimentos domiciliares, o Estado, ao determinar a política salarial com a qual tratará seus funcionários, afeta a desigualdade total no país. O impacto sobre a desigualdade do diferencial salarial público-privado, no entanto, é pequeno. A vantagem salarial dos funcionários públicos não é desprezível, mas tem um efeito reduzido na determinação da desigualdade total no Brasil. Se fosse considerada apenas a distribuição dos rendimentos do trabalho, esse impacto seria superior ao obtido para a desigualdade total.

A concentração dos rendimentos dos trabalhadores do setor público está fortemente associada a uma composição particular da força de trabalho nesse setor. Mesmo que não tivessem vantagens salariais e fossem remunerados de modo similar aos trabalhadores formais comparáveis do setor privado, esses trabalhadores tenderiam a ocupar os estratos mais altos da sociedade. Essa concentração é agravada pelo fato do diferencial salarial entre trabalhadores públicos e privados ser regressivo e altamente concentrado. Em uma estimativa mais conservadora o coeficiente de concentração na distribuição de rendimentos domiciliares per capita dos diferenciais positivos - isto é, dos prêmios salariais - alcança o nível de 0,863 , quando o coeficiente de Gini observado, já alto, é 0,540.

Apesar de muito concentrado, o diferencial salarial tem volume pequeno quando comparado às demais fontes de rendimentos. Consequentemente, sua contribuição para a desigualdade total é limitada. A participação do diferencial na renda domiciliar é estimada entre $1,4 \%$ e $1,8 \%$, valores muito baixos para que esse diferencial pudesse ter impactos expressivos sobre a distribuição dos rendimentos domiciliares. A estimativa é de que o diferencial salarial entre 
trabalhadores dos setores público e privado não contribua com mais de $3,1 \%$ da desigualdade total, medida pelo coeficiente de Gini da distribuição dos rendimentos domiciliares per capita. Já a decomposição de fatores da distribuição indica que a contribuição de outras fontes de rendimento para a desigualdade total é muito superior à contribuição dos diferenciais salariais. A distribuição observada dos rendimentos dos trabalhadores formais do setor privado contribuiria com algo em torno de $26,4 \%$ da desigualdade; os rendimentos dos empregados do setor público, já descontado o diferencial estimado, com 11,2\%. Consequentemente, o tamanho relativo e os efeitos de composição da força de trabalho nos dois setores são muito mais importantes para a desigualdade que os efeitos de segmentação do mercado de trabalho nesses dois setores.

Esses resultados têm algumas implicações para políticas que visam à redução das desigualdades sociais. Embora possa ser moralmente condenável que alguns funcionários públicos tenham salários expressivamente superiores aos que receberiam caso exercessem atividades semelhantes no setor privado, não se deve esperar que uma redução dessas vantagens tenha efeitos expressivos sobre a desigualdade total. Cortes de vantagens podem render dividendos políticos e, eventualmente, alinhamento eleitoral, mas não são cruciais para a redistribuição da renda no país. Por outro lado, aumentos reais dos salários do funcionalismo público tendem a elevar a desigualdade, ainda que pouco, pois as evidências são de que os trabalhadores do setor público pertençam a estratos superiores da distribuição de renda e recebam mais que os equivalentes do setor privado.

Os impactos aqui tratados limitam-se à análise da contribuição dos diferenciais para a desigualdade. Foge ao escopo do estudo analisar quais seriam os impactos sobre a desigualdade de um uso alternativo desses recursos. Os resultados obtidos não trazem nenhuma indicação nesse sentido. Como o diferencial total é positivo, sua redução implica liberação de recursos públicos que poderiam ser aplicados em outras atividades. Essa aplicação poderia ser equalizadora, concentradora ou neutra.

Também não é objetivo do estudo avaliar os resultados da redução dos diferenciais nos gastos com o funcionalismo público, mas, diferente do uso alternativo dos recursos, os resultados obtidos trazem algumas indicações de impacto direto. O diferencial salarial total 
estimado aqui representa algo em torno de $17 \%$ da massa salarial dos trabalhadores públicos. Uma redução desse diferencial tenderia a reduzir custos com o funcionalismo, mas seria apressado supor que essa redução poderia equivaler a esses $17 \%$. Parte dos menores salários do setor privado pode estar associada à discriminação racial e de gênero, fatores redutores de salários que não são aceitáveis em qualquer política salarial, menos ainda em uma política de Estado. Além disso, uma parte não desprezível dos menores salários do setor privado está relacionada à segmentação espacial do mercado de trabalho, com desvantagens para as regiões mais pobres. Para políticas de desenvolvimento regional talvez não seja sequer desejável que o Estado reforce desigualdades espaciais para atender metas de redução de custos com o funcionalismo. Finalmente, princípios de isonomia entre categorias profissionais são fatores adicionais que podem fazer com que o total de custos redutíveis do funcionalismo não seja alto.

Devido à segmentação do mercado, e diferenças nos objetivos e atividades de cada setor, é muito difícil estabelecer uma política salarial para trabalhadores do setor públicos afastada de controvérsias. Se é possível que algumas atividades no setor público tenham particularidades que justifiquem maiores salários, também se deve levar em conta que muitos desses trabalhadores já têm outras vantagens, algumas determinadas por lei, como um sistema previdenciário mais generoso e garantias de estabilidade, outras pela prática administrativa, como menor rigor no controle de produtividade. Deve ser considerada uma das limitações deste estudo o fato de ele ser insuficiente para guiar de modo seguro a determinação de uma política salarial desse tipo.

As conclusões substantivas deste estudo se mostraram robustas a modificações de definições de "trabalhador do setor público" e "trabalhador do setor privado equivalente", bem como a diferentes especificações de modelo para estimativa dos diferenciais. Alterações de resultados decorrentes da aplicação de técnicas de correção de viés de seleção, seja usando modelos probit simples ou modelos probit bivariados, não têm relevância. $\mathrm{O}$ mesmo pode ser dito da inclusão de variáveis de controle nas regressões para o pertencimento a categorias ocupacionais como profissionais das ciências e das artes, profissionais em geral, trabalhadores em serviços administrativos, técnicos de nível médio e trabalhadores do setor serviços. A exclusão 
dos militares do conjunto dos trabalhadores do setor público e dos trabalhadores na agricultura e nos serviços domésticos do conjunto dos empregados formais privados tem efeitos modestos nos resultados, os quais não modificam as conclusões substantivas.

Outra limitação do estudo é que nada assegura que as conclusões obtidas para o Brasil como um todo se manteriam em uma análise de unidades geográficas menores. Embora estudos anteriores tenham indicado que as vantagens salariais dos trabalhadores do setor público sejam maiores entre os servidores públicos federais, os quais se concentram em algumas unidades da federação, é possível que estados e municípios com elevadas proporções de funcionários públicos os impactos sobre a desigualdade da supressão dos diferenciais salariais sejam superiores aos estimados para o Brasil. Apenas uma análise específica para essas unidades permitiria dizer se esse é realmente o caso.

\section{Referências}

BELLUZZO, W., ANUATTI-NETO, F., \& PAZELLO, E. T. (2005). Distribuição de salários e o diferencial público-privado no Brasil. Revista Brasileira de Economia, 59(4), 511-533.

BENDER, S., \& FERNANDES, R. (2009). Gastos Públicos com Pessoal: Uma Análise de Emprego e Salário no Setor Público Brasileiro nos Anos 90. Revista EconomiA, 10(1), 19-47.

BRAGA, B. G. (2007). Capital Humano e o Diferencial de Salários Público-Privado no Brasil. Anais do XXXV Encontro Nacional de Economia [Proceedings of the 35th Brazilian Economics Meeting] (pp. 1-15). ANPEC-Associação Nacional dos Centros de Pós-graduação em Economia [Brazilian Association of Graduate Programs in Economics].

CHEN, C.-N., TSAUR, T.-W., \& RHAI, T.-S. (1982). The Gini Coefficient and Negative Income. Oxford Economic Papers, New Series, 34(3), 473-478.

FOGUEL, M. N., GILL, I., MENDONÇA, R., \& BARROS, R. P. de. (2000). The public-private wage gap in Brazil. Revista Brasileira de Economia, 54. doi:10.1590/S0034-71402000000400003

GREGORY, R. G., \& BORLAND, J. (1999). Recent developments in public sector labor markets. Handbook of labor economics, 3, 3573-3630.

HOFFMANN, R. (2009). Desigualdade da distribuição da renda no Brasil: a contribuição de aposentadorias e pensões e de outras parcelas do rendimento domiciliar per capita. Economia e Sociedade, $18(1), 213-231$.

HOLANDA, A. L. N. (2009). Diferencial de salários entre os setores público e privado : uma resenha da literatura. Brasília: IPEA.

HOLANDA-BARBOSA, A. L. N., BARBOSA-FILHO, FERNANDO H. (2012). Diferencial de salários entre os setores público e privado no Brasil : um modelo de escolha endógena. Brasília: IPEA.

JUHN, C., MURPHY, K. M., \& PIERCE, B. (1993). Wage inequality and the rise in returns to skill. Journal of political Economy, 410-442. 
MARCONI, N. (2003). A evolução do perfil da força de trabalho e das remunerações nos setores público e privado ao longo da década de 1990. Revista do Serviço Público, 54(1), 9-45.

PANIZZA, U., \& QIANG, C. Z. (2005). Public-private wage differential and gender gap in Latin America: Spoiled bureaucrats and exploited women? Journal of Socio-Economics, 34(6), 810-833.

PYATT, G., CHEN, C., \& FEI, J. (1980). The Distribution of Income by Factor Components. The Quarterly Journal of Economics, 95(3), 451-473. doi:10.2307/1885088.

RAO, V. M. (1969). Two Decompositions of Concentration Ratio. Journal of the Royal Statistical Society. Series A (General), 132(3), 418-425. doi:10.2307/2344120.

SHORROCKS, A. F. (1982). Inequality Decomposition by Factor Components. Econometrica, 50(1), 193-211. doi:10.2307/1912537

VAZ, D. V., \& HOFFMANN, R. (2007). Remuneração nos serviços no Brasil: o contraste entre funcionários públicos e privados. Economia e Sociedade, 16, 199-232.

VERGARA, D. H. (1991). Diferenciais de salários entre os setores publico e privado da economia brasileira. Ensaios FEE, 12(1), 73-8S.

VERGARA, D. H., \& DA SILVA WILTGEN, R. (1995). Os diferenciais de salários entre o setor público e o setor privado na RMPA. Indicadores Economicos FEE, 23(3).

\section{Anexo 1}

\section{Estimativas do Diferencial Salarial}

O modelo econométrico básico para estimar os salários dos trabalhadores do setor público e privado ( $w_{i} \mathrm{e} q_{i}$, respectivamente) com base em um vetor de variáveis explicativas $X$ é dado por:

$$
\begin{aligned}
& \ln \left(w_{i}\right)=X \beta_{w}+u_{w} \\
& \ln \left(q_{i}\right)=X \beta_{q}+u_{q}
\end{aligned}
$$

Um pressuposto deste modelo, que estima as duas equações em separado, é de que os trabalhadores são alocados de forma aleatória entre os dois setores. Caso contrário, há um viés de seleção, que pode afetar os coeficientes. Passa a ser importante, então, modelar o processo de seleção entre setores. Para isso, trata-se a variável dicotômica $I$, que assume valores assume valores 0 (setor privado) ou 1 (setor público), como uma variável latente $I^{*}$ dada por:

$$
I^{*}=Z \theta+\varepsilon
$$


Se $I^{*} \geq 0$, então $I=1$; se $I^{*}<0$, então $I=0$.

Com isso, pode-se montar o probit:

$\operatorname{Pr}(I=1 \mid Z)=\operatorname{Pr}\left(I^{*} \geq 0\right)=\operatorname{Pr}\left(\varepsilon<Z^{\prime} \theta\right)=\Phi\left(Z^{\prime} \theta\right)$

Onde $Z$ é um vetor de variáveis explicativas que inclui tanto as variáveis da equação de salários original quanto um conjunto específico de variáveis identificadoras da escolha de setor (restrições de exclusão).

As probabilidades preditas pelo Modelo (3) são então utilizadas para construir a razão inversa de Mills $\left(\lambda_{i}\right)$, que passa a ser incluída nas equações de rendimentos para controlar o viés de seleção:

$$
\begin{aligned}
& \ln \left(w_{i}\right)=X \beta_{w}+\lambda_{w}(Z \theta) \beta_{\lambda w}+u_{w} \\
& \ln \left(q_{i}\right)=X \beta_{q}+\lambda_{q}(-Z \theta) \beta_{\lambda q}+u_{q}
\end{aligned}
$$

Uma limitação desse modelo probit simples é que ele só leva em conta a escolha do setor, condicional à decisão anterior de trabalhar ou não. Para dar conta desses dois possíveis vieses de seleção - gerados pela decisão de trabalhar ou não e pela escolha do setor - empregamos nas simulações mais complexas um modelo de probit bivariado e construímos duas razões inversas de Mills baseadas nas previsões lineares.

Holanda-Barbosa e Barbosa-Filho (2012) usam um procedimento de correção mais sofisticado, baseado em modelos de regressão com mudança endógena para tratar o viés de seleção de setores. Esses modelos são estimados separadamente por gênero. No entanto, concluem que esse procedimento subestima o diferencial salarial entre as mulheres devido, possivelmente, a problemas de especificação das variáveis que tentam captar características não observáveis das trabalhadoras. Analisando os resultados de Holanda-Barbosa e BarbosaFilho é possível dizer que a adoção de sua estratégia de correção de viés levaria a conclusões substantivas semelhantes às que chegamos.

Uma vez obtida as equações de rendimentos apropriadas para os setores público e privado, com ou sem correção para viés de seleção, 
o passo seguinte foi construir a renda contrafactual dos funcionários públicos. Para isso, empregamos a decomposição proposta por Juhn, Murphy e Pierce (1993). Considere, primeiro, que o resíduo da equação de rendimentos dos trabalhadores do setor privado pode ser desagregado em dois componentes: a posição do indivíduo na distribuição dos resíduos $\left(\tau_{i q}\right)$ e a função de distribuição dos resíduos $\left(F_{q}\right)$. Logo:

$$
u_{q}=F_{q}^{-1}\left(\tau_{\dot{q}} \mid X\right)
$$

Onde $F_{q}^{-1}(. \mid X)$ é a inversa da função de distribuição acumulada

para trabalhadores do setor privado com características $X$. Com isso, os rendimentos contrafactuais $\left(c f_{i}\right)$ dos funcionários públicos podem ser estimados por:

$$
\ln \left(c f_{i}\right)=X \beta_{q}+F_{q}^{-1}\left(\tau_{i w} \mid X\right)
$$

Ou seja, a renda contrafactual é calculada mantendo os mesmos coeficientes obtidos para os trabalhadores do setor privado e a mesma função de distribuição dos resíduos. Para mais detalhes, ver Juhn, Murphy e Pierce (1993).

\section{Decomposição por Fatores do Índice de Gini}

Considere uma população com $n$ indivíduos de renda $x_{i}$ e renda média igual $\mu$. Se ordenarmos todos os indivíduos da menor para a maior renda $\left(x_{1} \leq x_{2} \leq \ldots \leq x_{n}\right)$, atribuindo a cada um uma posição $i$ no ordenamento, então o índice de Gini pode ser escrito como:

$$
G=\frac{2}{n \mu} \operatorname{cov}\left(i, x_{i}\right)
$$

Se a renda $x_{i}$ puder ser expressa como a soma da renda de $k$ fatores componentes, então:

$$
x_{i}=\sum_{h=1}^{k} x_{h i}
$$




$$
G=\sum_{h=1}^{k} \phi_{h} C_{h}
$$

Onde $\phi_{h}$ é a fração da renda $h$ sobre a renda total e $C_{h}$ é o coeficiente de concentração da renda $h$, dado por:

$$
C_{h}=\frac{2}{n} \operatorname{cov}\left(i, \frac{x_{h i}}{\mu_{h}}\right)
$$

Logo, a contribuição absoluta de cada fonte $\mathrm{h}$ para a desigualdade total é dada por $\phi_{h} C_{h}$ e a contribuição relativa é igual $\left(\phi_{h} C_{h}\right) / G$. Para mais detalhes, ver Shorrocks (1982). 